\section{Logical Document Structure}

Thijs Westerveld

Teezir Search Solutions, Ede, Netherlands

\section{Definition}

Logical structure refers to the way information in a document is organized; it defines the hierarchy of information and the relation between different parts of the document. Logical structure indicates how a document is built, as opposed to what a document contains.

\section{Key Points}

Logical structure is mainly used in the context of XML document to distinguish the logical organization of the content from its physical organization, to distinguish the flow of content from the documents layout and from the presentation of the document. In XML documents, text, images and metadata can be organized in a meaningful, logical manner independently of the document's layout when presented to a user. This contrasts with HTML documents in which logical organization and layout necessarily are the same. The logical structure of a document is of particular interest in structured document retrieval, where it may provide knowledge regarding the organization of information which may lead to better identification of relevant document parts given a user's request.

\section{Cross-References}

- Processing Structural Constraints

- Structured Text Models

- XML Retrieval 